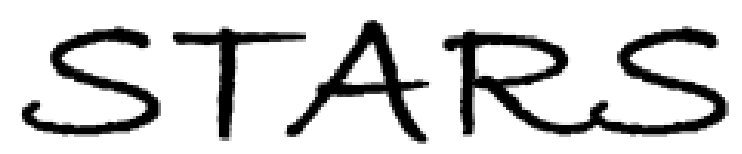

University of Central Florida

STARS

$1-1-1999$

\title{
Magnetohydrodynamic boundary layer on a flat plate: Further analytic results
}

\author{
Bhimsen K. Shivamoggi \\ University of Central Florida \\ David K. Rollins \\ University of Central Florida
}

Find similar works at: https://stars.library.ucf.edu/facultybib1990

University of Central Florida Libraries http://library.ucf.edu

This Article is brought to you for free and open access by the Faculty Bibliography at STARS. It has been accepted for inclusion in Faculty Bibliography 1990s by an authorized administrator of STARS. For more information, please contact STARS@ucf.edu.

\section{Recommended Citation}

Shivamoggi, Bhimsen K. and Rollins, David K., "Magnetohydrodynamic boundary layer on a flat plate: Further analytic results" (1999). Faculty Bibliography 1990s. 2851.

https://stars.library.ucf.edu/facultybib1990/2851

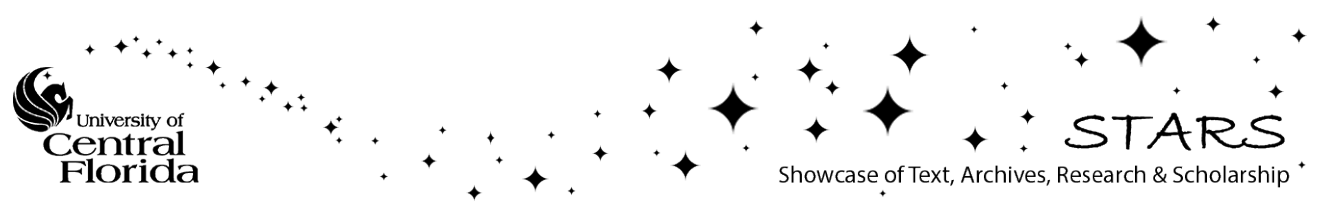




\section{Magnetohydrodynamic boundary layer on a flat plate: Further analytic results}

Cite as: Journal of Mathematical Physics 40, 3372 (1999); https://doi.org/10.1063/1.532893

Submitted: 02 June 1998 . Accepted: 04 March 1999 . Published Online: 22 June 1999

Bhimsen K. Shivamoggi, and David K. Rollins

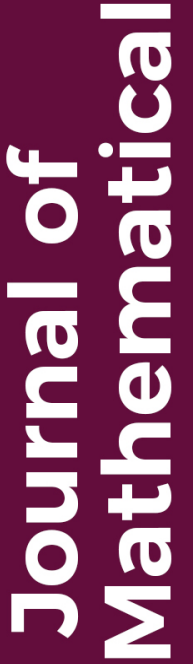

\section{AVS Quantum Science}




\title{
Magnetohydrodynamic boundary layer on a flat plate: Further analytic results
}

\author{
Bhimsen K. Shivamoggi and David K. Rollins ${ }^{\mathrm{a})}$ \\ Department of Mathematics, University of Central Florida, Orlando, Florida 32816
}

(Received 2 June 1998; accepted for publication 4 March 1999)

Further analytic results are deduced with the magnetohydrodynamic boundary layer equations for a flat plate. The asymptotic behavior of the solutions is deduced using the scaling group method. Then, an analytic perturbative procedure is used to determine an approximate solution that exhibits this asymptotic behavior. (C) 1999 American Institute of Physics. [S0022-2488(99)02906-0]

\section{INTRODUCTION}

Greenspan and Carrier ${ }^{1}$ considered the flow of a viscous, electrically conducting, incompressible fluid past a semi-infinite flat plate in the presence of a magnetic field which is uniform at infinity and parallel to the stream. They reduced the boundary-layer equations then to a pair of coupled nonlinear ordinary differential equations-

$$
\begin{aligned}
& g^{\prime \prime}+\mu\left(f g^{\prime}-f^{\prime} g\right)=0, \\
& f^{\prime \prime \prime}+f f^{\prime \prime}-\frac{1}{A^{2}} g g^{\prime \prime}=0,
\end{aligned}
$$

subject to the boundary conditions

$$
\begin{gathered}
\eta=0: f=0, \quad f^{\prime}=0, \quad g=0, \\
\eta \Rightarrow \infty: f \approx 2 \eta, \quad g \approx 2 \eta .
\end{gathered}
$$

Here, primes denote differentiation with respect to the independent variable $\eta=\frac{1}{2} y \sqrt{U / \nu x} ; y$ measures the distance from the plate, $x$ is the distance along the plate from the leading edge, $U$ is the undisturbed velocity, and $\nu$ is the kinematic viscosity. Further, if $u$ and $B_{x}$ are the $x$ components of the velocity and magnetic fields, then

$$
u=\frac{1}{2} U f^{\prime}(\eta), \quad B_{x}=\frac{1}{2} B_{0} g^{\prime}(\eta)
$$

$B_{0}$ being the ambient magnetic field intensity. Finally, $A \equiv U / V_{\mathrm{A}}$, where $V_{\mathrm{A}}$ is the Alfvén velocity $V_{\mathrm{A}} \equiv B_{0} / \sqrt{4 \pi \rho}$, and $\mu \equiv 4 \pi \sigma \nu, \rho$ being the density and $\sigma$ the electrical conductivity of the fluid.

For sub-Alfvénic flows $(A<1)$, disturbances travel upstream of the plate, invalidating the notion of a boundary layer originating at the leading edge of the plate. As Greenspan and Carrier ${ }^{1}$ pointed out, this can be clearly seen by considering the case with infinite electrical conductivity, $\mu \Rightarrow \infty$. For this case, Eqs. (1) and (2) become $g=f, f^{\prime \prime \prime}+\left(1+\left(1 / A^{2}\right)\right) f f^{\prime \prime}=0$, so that one needs $A^{2}>1$ in order to preserve the usual boundary-layer situation. Reuter and Stewartson ${ }^{2}$ showed that, for this case, the problem is mathematically ill posed, in the sense that it does not admit any solutions such that $f^{\prime \prime}(0)>0$ and $g^{\prime}(0)>0$. Stewartson and Wilson ${ }^{3}$ showed further that, even for certain values of $A>1$, the solutions turn out to be nonunique whenever $\mu<1$.

${ }^{a)}$ Electronic mail: drollins@ pegasus.cc.ucf.edu 
Equations (1) and (2) are, on the other hand, highly nonlinear and, therefore, one may not anticipate explicit analytical solutions for them. In this paper, we first deduce the asymptotic behavior of the solutions of Eqs. (1) and (2) using the scaling group method (Bluman and Kumei $\left.^{4}\right)$. We then use an analytical perturbative procedure due to Bender et $a l^{5}$ to determine an approximate solution that has the above asymptotic behavior.

\section{ASYMPTOTIC BEHAVIOR OF THE SOLUTION}

In order to find the asymptotic behavior of the solutions of (1)-(3), note first that Eqs. (1) and (2) admit solutions of the form

$$
f \sim \frac{1}{\eta}, \quad g \sim \frac{1}{\eta}
$$

(4) implies that Eqs. (1) and (2) have the scaling group

$$
\bar{f}=\alpha^{-1} f, \quad \bar{g}=\alpha^{-1} g, \quad \bar{\eta}=\alpha \eta .
$$

We may therefore introduce the following canonical coordinates

$$
s=f \eta, \quad t=\eta^{2} \frac{d f}{d \eta}, \quad q=\eta g .
$$

The transformation from $(s, t)$ to $(f, \eta)$ is given differentially by

$$
\frac{d s}{t+s}=\frac{d \eta}{\eta}
$$

The transformation rules of the various derivatives are

$$
\begin{gathered}
\frac{d^{2} f}{d \eta^{2}}=-\frac{2 t}{\eta^{3}}+\frac{1}{\eta^{3}}(t+s) \frac{d t}{d s} \\
\frac{d^{3} f}{d \eta^{3}}=\frac{6 t}{\eta^{4}}-\frac{5}{\eta^{4}}(t+s) \frac{d t}{d s}+\frac{1}{\eta^{4}}(t+s)^{2} \frac{d^{2} t}{d s^{2}}+\frac{1}{\eta^{4}}(t+s) \frac{d t}{d s}\left(\frac{d t}{d s}+1\right) \\
\frac{d g}{d \eta}=-\frac{q}{\eta^{2}}+\frac{1}{\eta^{2}}(t+s) \frac{d q}{d s} \\
\frac{d^{2} g}{d \eta^{2}}=\frac{2}{\eta^{3}} q-\frac{3}{\eta^{3}}(t+s) \frac{d q}{d s}+\frac{1}{\eta^{3}}(t+s)^{2} \frac{d^{2} q}{d s^{2}}+\frac{1}{\eta^{3}}(t+s) \frac{d q}{d s}\left(\frac{d t}{d s}+1\right)
\end{gathered}
$$

In terms of the new variables $(s, t, q)$, the boundary-value problem (1)-(3) becomes

$$
\begin{gathered}
6 t-5(t+s) \frac{d t}{d s}+(t+s)^{2} \frac{d^{2} t}{d s^{2}}+(t+s) \frac{d t}{d s}\left(\frac{d t}{d s}+1\right)-2 s t+s(t+s) \frac{d t}{d s} \\
-\frac{1}{A^{2}} q[2 q-3(t+s)] \frac{d q}{d s}+(t+s)^{2} \frac{d^{2} q}{d s^{2}}+(t+s) \frac{d q}{d s}\left(\frac{d t}{d s}+1\right)=0, \\
2 q-3(t+s) \frac{d q}{d s}+(t+s)^{2} \frac{d^{2} q}{d s^{2}}+(t+s) \frac{d q}{d s}\left(\frac{d t}{d s}+1\right)+\mu\left[s(t+s) \frac{d q}{d s}-q(t+s)\right]=0, \\
s=0: t=0, \quad q=0,
\end{gathered}
$$




$$
s \Rightarrow \infty: t \Rightarrow \infty, \quad q \Rightarrow \infty
$$

Near $s=0$, Eqs. (9) and (10) show that

$$
t \approx \lambda_{1} s, \quad q \approx \lambda_{2} s^{p},
$$

with

$$
\begin{gathered}
6 \lambda_{1}-5 \lambda_{1}\left(\lambda_{1}+1\right)+\lambda_{1}\left(\lambda_{1}+1\right)^{2} \approx 0, \\
2 \lambda_{2}-3 p \lambda_{2}\left(\lambda_{1}+1\right)+p(p-1) \lambda_{2}\left(\lambda_{1}+1\right)^{2} \lambda_{2} p\left(\lambda_{1}+1\right)^{2} \approx 0,
\end{gathered}
$$

or

$$
\lambda_{1}=1,2,
$$

$$
\lambda_{1}=2, \lambda_{2} \text { arbitrary and } p=\frac{1}{3}, \frac{2}{3} \text {. }
$$

$\lambda_{1}=1$ turns out to be the spurious root. The root $p=\frac{1}{3}$ is to be discarded because we require from (6) and (13) that $2 p>1$.

For $\lambda_{1}=2$, we obtain from (7) and (13),

$$
s \sim \eta^{3}
$$

Using (16), we have from (6),

$$
\eta \Rightarrow 0: f \sim \eta^{2}, \quad g \sim \eta
$$

Near $s \Rightarrow \infty$, equations (9) and (10) show that

$$
t \approx \tilde{\lambda_{1}} s, \quad q \approx \tilde{\lambda_{2}} s
$$

with

$$
\begin{aligned}
& -2 \tilde{\lambda_{1}}+\tilde{\lambda_{1}}\left(\tilde{\lambda_{1}}+1\right)-\frac{1}{A^{2}} \tilde{\lambda_{2}}\left[2 \tilde{\lambda_{2}}-3 \tilde{\lambda_{2}}\left(\tilde{\lambda_{1}}+1\right)+\tilde{\lambda_{2}}\left(\tilde{\lambda_{1}}+1\right)^{2}\right] \approx 0, \\
& 2 \tilde{\lambda_{2}}-3 \tilde{\lambda_{2}}\left(\tilde{\lambda_{1}}+1\right)+\tilde{\lambda_{2}}\left(\tilde{\lambda_{1}}+1\right)^{2} \approx 0
\end{aligned}
$$

or

$$
\begin{gathered}
\tilde{\lambda_{1}}=1, \\
\tilde{\lambda_{2}} \text { arbitrary. }
\end{gathered}
$$

Using (19) and (20), we obtain from (7) and (18),

$$
s \sim \eta^{2}
$$

Using (21), we have from (6),

$$
\eta \Rightarrow \infty: f \sim \eta, \quad g \sim \eta
$$

Observe that the asymptotic behavior of the solutions, as exhibited by (17) and (22), is independent of the Alfvén number $A$. 


\section{AN ANALYTIC PERTURBATIVE SOLUTION}

We now use a perturbative procedure due to Bender et $a l .{ }^{5}$ to solve Eqs. (1) and (2) analytically. This method has been used recently (Shivamoggi and Rollins ${ }^{6}$ ) to solve the Kadomtsev equation for a heavy atom in a very strong magnetic field with very good results. We first replace Eqs. (1) and (2) by ones that contain a parameter $\delta$, i.e.,

$$
\begin{aligned}
& g^{\prime \prime}+\mu\left[g^{\prime} f^{\delta}-f^{\prime} g^{\delta}\right]=0, \\
& f^{\prime \prime \prime}+f^{\prime \prime} f^{\delta}-\frac{1}{A^{2}} g^{\prime \prime} g^{\delta}=0 .
\end{aligned}
$$

Note that Eqs. (1) and (2) are recovered when $\delta=1$, and $\delta=0$ corresponds to the linear zerothorder approximation. By identifying $\delta$ as the perturbation parameter, the solution $(f, g)$ is then expanded in a power series in $\delta$,

$$
\begin{aligned}
& f=f_{0}+\delta f_{1}+\delta^{2} f_{2}+\cdots, \\
& g=g_{0}+\delta g_{1}+\delta^{2} g_{2}+\cdots .
\end{aligned}
$$

This then leads to a set of linear equations for $\left(f_{n}, g_{n}\right): O(1)$,

$$
\begin{aligned}
& g_{0}^{\prime \prime}+\mu\left(g_{0}^{\prime}-f_{0}^{\prime}\right)=0, \\
& f_{0}^{\prime \prime \prime}+f_{0}^{\prime \prime}-\frac{1}{A^{2}} g_{0}^{\prime \prime}=0 ;
\end{aligned}
$$

$O(\delta)$,

$$
\begin{aligned}
& g_{1}^{\prime \prime}+\mu\left(g_{1}^{\prime}-f_{1}^{\prime}\right)=-\mu\left(g_{0}^{\prime} \cdot \ln f_{0}-f_{0}^{\prime} \cdot \ln g_{0}\right), \\
& f_{1}^{\prime \prime \prime}+f_{1}^{\prime \prime}-\frac{1}{A^{2}} g_{1}^{\prime \prime}=-f_{0}^{\prime \prime} \cdot \ln f_{0}+\frac{1}{A^{2}} g_{0}^{\prime \prime} \cdot \ln g_{0},
\end{aligned}
$$

etc.

Successive integrations of Eqs. (26) and (27), along with the use of (3), lead to

$$
\begin{aligned}
& g_{0}^{\prime}+\mu\left(g_{0}-f_{0}\right)=\alpha, \\
& f_{0}^{\prime}+f_{0}-\frac{1}{A^{2}} g_{0}=c \eta,
\end{aligned}
$$

where $c$ is an arbitrary constant and

$$
\alpha \equiv g_{0}^{\prime}(0)>0 .
$$

Using the boundary condition (3) at $\eta \Rightarrow \infty$, we obtain, from (31),

$$
c=2 \epsilon,
$$

where

$$
\epsilon \equiv 1-\frac{1}{A^{2}}>0
$$

We have, from Eqs. (30) and (31), 


$$
f_{0}^{\prime \prime}+(1+\mu) f_{0}^{\prime}+\epsilon \mu f_{0}=2 \epsilon \mu \eta+2 \epsilon+\alpha(1-\epsilon),
$$

from which

$$
f_{0}=\left(D_{1} e^{\sigma_{1} \eta}+D_{2} e^{\sigma_{2} \eta}\right)-\frac{2 \mu+(2-\alpha)(1-\epsilon)}{\mu \epsilon}+2 \eta,
$$

where

$$
\sigma_{1,2}=\frac{1}{2}\left[-(1+\mu) \pm \sqrt{(1+\mu)^{2}-4 \mu \epsilon}\right] .
$$

Using the boundary condition (3) at $\eta=0$, we obtain

$$
\begin{gathered}
D_{1}=-\frac{1}{\sigma_{1}-\sigma_{2}}\left[\sigma_{2} \frac{2 \mu+(2-\alpha)(1-\epsilon)}{\mu \epsilon}+2\right], \\
D_{2}=\frac{1}{\sigma_{1}-\sigma_{2}}\left[\sigma_{1} \frac{2 \mu+(2-\alpha)(1-\epsilon)}{\mu \epsilon}+2\right] .
\end{gathered}
$$

We have from (32) and (35) and (36), for small $\eta$,

$$
\begin{gathered}
f_{0} \approx \frac{1}{2}[\alpha(1-\epsilon)+2 \epsilon] \eta^{2}, \\
g_{0} \approx \alpha \eta .
\end{gathered}
$$

One may also obtain (37) directly from Eqs. (30) and (31).

Observe from (37a) that, for super-Alfvénic flows $(\epsilon>0), f_{0}^{\prime \prime}(0)>0$, because

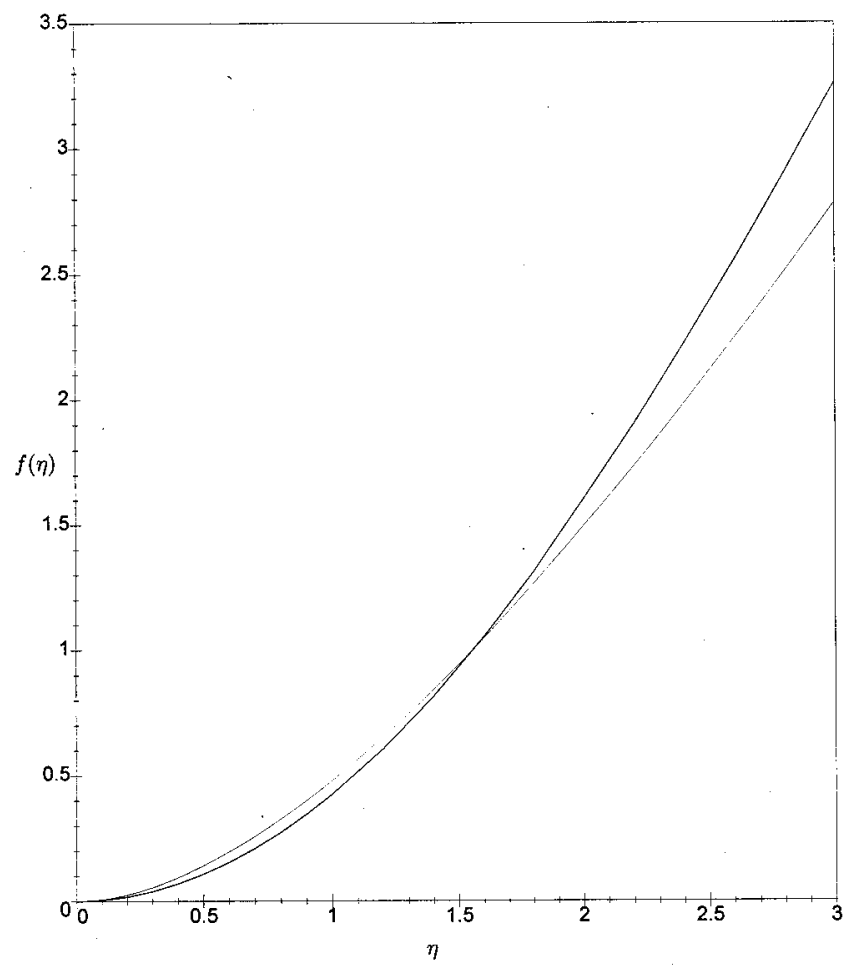

FIG. 1. Comparison of zeroth order approximate solution and numerical solution (bold) for nonlinear boundary value problem for magnetohydrodynamic case. 


$$
\alpha(1-\epsilon)+2 \epsilon>0
$$

on noting (32) and that $(1-\epsilon)>0$.

Further, we have from (31), (33), and (35), for large $\eta$,

$$
\begin{aligned}
& f_{0} \approx 2 \eta, \\
& g_{0} \approx 2 \eta .
\end{aligned}
$$

One may also obtain (38) directly from Eqs. (30) and (31).

The agreement of (37) with (17) on the one hand, and (38) with (3b) on the other hand, indicates that the asymptotic behavior (for both small and large $\eta$ ) of the solution of Eqs. (1) and (2) can be accurately provided by the linearized versions of the latter. Indeed, the linearized (or the zeroth-order) solution turns out to provide a reasonably accurate representation of the exact numerical solution of Eqs. (1) and (2) elsewhere as well.

In Fig. 1, the zeroth-order approximate analytic solution $f_{0}$ given in (35) is compared with the exact numerical solution of Eqs. (1) and (2). The agreement seems to be very good, even though $f_{0}$ is meant to be only a crude approximation to the exact solution. In fact, this feature is a carryover from the hydrodynamic case. In the latter case, (35) reduces to

$$
f_{0}(\eta)=2 e^{-\eta}-2+2 \eta
$$

in agreement with the one given by Bender $e t$ al. ${ }^{5}$ In Fig. 2, the zeroth-order approximate analytic solution given by (39) is compared with the exact numerical solution of the Blasius equation,

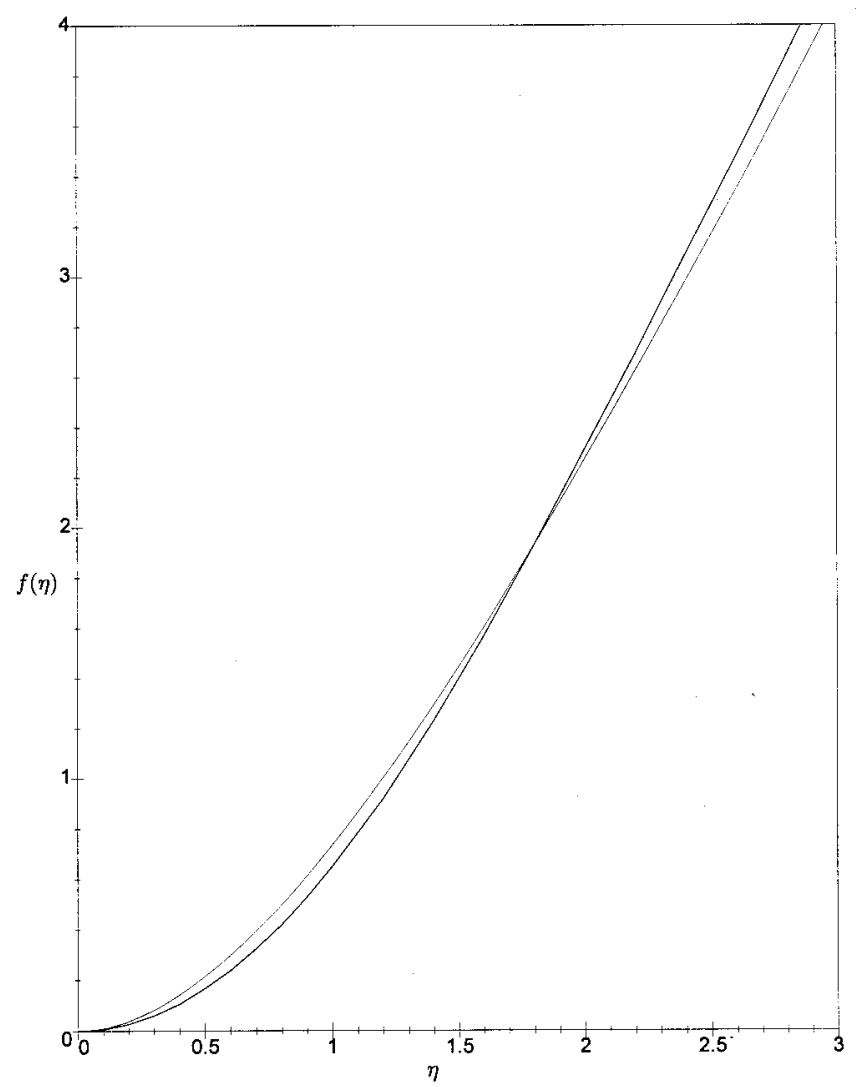

FIG. 2. Comparison of zeroth order approximate solution and numerical solution (bold) for nonlinear boundary value problem for hydrodynamic case. 


$$
f^{\prime \prime \prime}+f f^{\prime \prime}=0
$$

Again, the agreement seems to be very good.

Next, using (31) and (35) in the $O(\delta)$ equations (28) and (29), we see that a closed-form analytic solution of these equations becomes very difficult to find.

\section{DISCUSSION}

In this paper, we have deduced further analytic results with the magnetohydrodynamic boundary layer equations (1) and (2) for a flat plate. We first derived the asymptotic behavior of the solutions using the scaling group method (Bluman and $\mathrm{Kumei}^{4}$ ). We then sought to use an analytic perturbative procedure due to Bender et $a .^{5}$ to determine an approximate solution. However, the linearized (or the zeroth-order) solution of the boundary-layer equations (1) and (2) turned out to provide not only the required asymptotic behavior (for both small and large $\eta$ ) of the exact numerical solution of Eqs. (1) and (2), but also a reasonably accurate representation of the exact numerical solution elsewhere.

\section{ACKNOWLEDGMENTS}

The authors are indebted to the referee for his valuable remarks that contributed to making the presentation of the material more accurate.

${ }^{1}$ H. P. Greenspan and G. F. Carrier, J. Fluid Mech. 6, 77 (1959).

${ }^{2}$ G. E. H. Reuter and K. Stewartson, Phys. Fluids 4, 276 (1961).

${ }^{3}$ K. Stewartson and D. H. Wilson, J. Fluid Mech. 18, 337 (1964).

${ }^{4}$ G. W. Bluman and S. Kumei: Group Symmetries and Differential Equations (Springer-Verlag, Berlin, 1989).

${ }^{5}$ C. M. Bender, K. A. Milton, S. S. Pensky, and L. M. Simmons, Jr., J. Math. Phys. 30, 1447 (1989).

${ }^{6}$ B. K. Shivamoggi and D. K. Rollins, J. Phys. A 30, 3681 (1997). 